\title{
Focusing on thyroid nodules in suspense: 10-15 mm with repeat cytology, Category III, the Bethesda System for Reporting Thyroid Cytopathology, TBSRTC
}

\author{
Ilker Sengul1,2 (1D, Demet Sengul ${ }^{3 *}$
}

\section{Dear Editor,}

Different management guidelines for thyroid nodules have been striving to enhance the diagnostic performance and to avoid unnecessary biopsy rates beyond surgery by determinants the different thyroid size thresholds, used as cutoff values, which are determinants for the scheduling of a thyroid nodule to undergo intervention of fine-needle aspiration (FNA). Ha et al. ${ }^{1}$ stated that the high specificity and low rate of unnecessary biopsies in the 2017 American College of Radiology (ACR) guidelines arise from larger cutoffs (mildly suspicious nodules, $25 \mathrm{~mm}$; moderately suspicious nodules, $15 \mathrm{~mm}$ ), as compared to the 2015 American Thyroid Association (ATA) Management Guidelines for Adult Patients with Thyroid Nodules and Differentiated Thyroid Cancer and the 2016 Korean Thyroid Association/ Korean Society of Thyroid Radiology (KTA/KSThR) guidelines (15 and $10 \mathrm{~mm}$, respectively). In addition, they denoted that the ATA and KTA/KSThR guidelines were exhibiting high sensitivity, whereas the ACR guideline was less significant in terms of the diagnostic performance of ultrasonography (US)-guided FNA for the indicated thyroid nodules. Therefore, they pointed out in their respectable paper that the thyroid nodules with low or intermediate suspicion for the 2015 ATA and 2016 KTA/ KSThR guidelines had undergone FNA at a cutoff of 25 and 15 $\mathrm{mm}$, respectively, which could produce significant changes in the percentage of thyroid nodules that needed an FNA application.

However, we have recently investigated whether the cutoff points of 10 and $15 \mathrm{~mm}$ are effective in three diagnostic tools: SE, US-guided FNA (US-FNA) cytology, and histopathology. These current outcomes based on the size, both above 10 and $15 \mathrm{~mm}$, revealed no significance regarding Tsukuba elasticity score (TES) $4 \& 5$ with the curves of the receiver operating characteristics
(ROC), the areas under the curves (AUCs) of 0.531 and 0.623 , respectively, the AUC of TES $4 \& 5$ above 15 was higher than above 10 . Therefore, it may be alleged that the possible correlation between the size and the TES $4 \& 5$ becomes stronger when the cutoff was designated to have $15 \mathrm{~mm}$. Likewise, the McNemar test was used to compare the nodules above 10 and $15 \mathrm{~mm}$ with malignant histopathology, and no significance was revealed with an additional calculation of the AUC of 0.509 and 0.515 , respectively. Hence, it may be concluded that the association between the nodule size and the precise histopathology increased as the cutoff point was raised $15 \mathrm{~mm}^{2}$, which is similar to the study of Ha et al ${ }^{1}$.

Nevertheless, a cutoff of $10 \mathrm{~mm}$ could still not be an underestimating issue for the management of the thyroid nodules to date ${ }^{3}$. Therefore, some authorities have been set at a cutoff value of $10 \mathrm{~mm}$ on the size selection criteria for US-FNA till today. For instance, the American Association of Clinical Endocrinologists (AACE)/Associazione Medici Endocrinologi (Italian Association of Clinical Endocrinologists) (AME) recommended FNA for the ones above $10 \mathrm{~mm}$, solid and hypoechoic in the US [Grade B; BEL (best evidence level) 3]. Besides, the novel European Thyroid Imaging and Reporting Data System (EU-TIRADS), based on a literature review and on the AACE, ATA, and Korean guidelines, comprising a thyroid US lexicon, proposed FNA for the nodules $>10 \mathrm{~mm}$ of a high-risk category (EU-TIRADS 5). In addition, the Society of Radiologists in Ultrasound (SRU) ${ }^{4}$ strongly suggested it for the ones $\geq 10 \mathrm{~mm}$ with microcalcifications. In addition, the 2009 ATA Management Guidelines for Adult Patients with Thyroid Nodules and Differentiated Thyroid Cancer recommended it for the ones $\geq 10 \mathrm{~mm}$ with microcalcifications and solid hypoechoic nodules, while the 2015 ATA Management Guidelines suggested the FNA for the nodules $\geq 10 \mathrm{~mm}$ with high-to-intermediate

'Giresun University, Faculty of Medicine, Endocrine Surgery Division - Giresun, Turkey.

${ }^{2}$ Giresun University, Faculty of Medicine, General Surgery Department - Giresun, Turkey.

${ }^{3}$ Giresun University, Faculty of Medicine, Pathology Department - Giresun, Turkey.

*Corresponding author: demet.sengul.52@gmail.com

Conflicts of interest: the authors declare there are no conflicts of interest. Funding: none.

Received on September 30, 2020. Accepted on October 18, 2020 
suspicion sonographic pattern (Recommendation 8I(A); Strong recommendation, Moderate-quality evidence, Recommendation 8I(B); Strong recommendation, Low-quality evidence, respectively).

The recommendation of the 2015 ATA Management Guidelines for the nodules $\geq 10 \mathrm{~mm}$ is obvious when stating that: it considers US-FNA for a high-to-intermediate suspicion sonographic pattern (Recommendation 8I(A); Strong recommendation, Moderate-quality evidence, Recommendation 8I(B); Strong recommendation, Lowquality evidence, respectively). However, the real challenging point is being able to decide as far as irreproachable management of the ones with controversial cytology, such as atypia of undetermined significance or follicular lesion of undetermined significance, AUS/FLUS, of indeterminate cytology. The 2015 ATA Management Guidelines recommend repeat FNA and molecular testing for a panel of mutations (BRAF, NRAS, HRAS, KRAS, RET/PTC1, RET/PTC3, PAX8/ $P P A R c)$ to supplement malignancy risk assessment in lieu of proceeding directly with a strategy of either surveillance or diagnostic surgery for (AUS/FLUS) indeterminate cytology (III of III, IV, and V, TBSRTC, $1^{\text {st }}$ and $2^{\text {nd }} \mathrm{ed}$ ) (Recommendation 15(A); Weak recommendation, Moderate-quality evidence). The last ATA Management Guidelines also suggest either surveillance or diagnostic surgical excision for an AUS/FLUS thyroid nodule, depending on the clinical risk factors, the sonographic pattern, and the patient's preference as repeated FNA cytology, molecular testing, or both, are not performed or inconclusive without specifying any cutoff size for nodules (Recommendation 15(B); Strong recommendation, Low-quality evidence).
Notably, the 2015 ATA Management Guidelines strongly recommended it, but with low-quality evidence. We have recently reported that the largest cutoff of $15 \mathrm{~mm}$ may strengthen its association with malignant histopathology, as compared to the lowest, of $10 \mathrm{~mm}^{2}$, as shown by some authors ${ }^{1}$. Therefore, in this study, we would like to open a new window regarding the management of the nodules, between 10 and $15 \mathrm{~mm}$ in the largest diameter with Category III, The Bethesda System for Reporting Thyroid Cytopathology (TBSRTC) of indeterminate cytology $y^{47}$, as repeated FNA cytology, molecular testing ${ }^{8}$, or both, are not performed or are inconclusive, and clinical risk factors, sonographic pattern ${ }^{4}$, and patient's preference are controversial. We recommend surveillance for the management of the mentioned thyroid nodules, $10-15 \mathrm{~mm}$ with Category III of indeterminate cytology, TBSRTC, 1st and 2nd ed., harboring high-to-intermediate suspicion sonographic pattern, low clinical risk factors, repeatead FNA cytology, molecular testing, or both, are not performed or inconclusive, comparing the ones $>15 \mathrm{~mm}$. This issue deserves further investigation. We hope we might contribute and provide new insights into that challenging window in Thyroidology.

\section{AUTHORS" CONTRIBUTIONS}

IS: Conceptualization, Formal Analysis, Writing - Review \& Editing. DS: Conceptualization, Formal Analysis, Investigation, Supervision Writing - Review \& Editing.

\section{REFERENCES}

1. Ha SM, Baek JH, Na DG, Suh CH, Chung SR, Choi YJ, et al. Diagnostic performance of practice guidelines for thyroid nodules: thyroid nodule size versus biopsy rates. Radiology. 2019;291(1):92-9. https://doi.org/10.1148/ radiol.2019181723

2. Sengul D, Sengul I, Egrioglu E, Ozturk T, Aydın I, Kesicioglu T, et al. Can cut-off points of 10 and $15 \mathrm{~mm}$ of thyroid nodule predict malignancy on the basis of three diagnostic tools: i) strain elastography, ii) the Bethesda System for Reporting Thyroid Cytology with 27-gauge fine-needle, and iii) histopathology? J BUON. 2020:25(2):1122-9. PMID: 32521915

3. Sengul I, Sengul D. Notes on "elastography for the diagnosis of high-suspicion thyroid nodules based on the 2015 American Thyroid Association guidelines: a multicenter study". North Clin Istanb. 2020;8(1):109-10. https://doi.org/10.14744/ nci.2020.74240

4. Frates MC, Benson CB, Charboneau JW, Cibas ES, Clark OH, Coleman BG, et al. Management of thyroid nodules detected at US: Society of Radiologists in Ultrasound consensus conference statement. Radiology. 2005;237(3):794-800. https://doi. org/10.1148/radiol.2373050220
5. Sengul D, Sengul I, Van Slycke S. Risk stratification of the thyroid nodule with Bethesda indeterminate cytology, category III, IV, V on the one surgeon-performed US-guided fine-needle aspiration with 27-gauge needle, verified by histopathology of thyroidectomy: The additional value of one surgeon-performed elastography. Acta Chir Belg. 2019;119(1):38-46. https://doi. org/10.1080/00015458.2018.1551769

6. Sengul D, Sengul I. Association between Tsukuba elasticity scores 4 and 5 on elastography and Bethesda undetermined cytology on US-guided FNA with 27-G needle, verified by histopathology: a cut-off point of $20 \mathrm{~mm}$ of diameter designated for thyroid nodules. J BUON. 2019;24(1):382-90. PMID: 30941995

7. Sengul D, Sengul I, Pelikán A. Paraphrase for the impact of repeat fine-needle aspiration in thyroid nodules categorized as atypia of undetermined significance or follicular lesion of undetermined significance: a single center experience. Diagn Cytopathol. 2021;49(3):452-3. https://doi.org/10.1002/dc.24685

8. Sponziello M, Brunelli C, Verrienti A, Grani G, Pecce V, Abballe $L$, et al. Performance of a dual-component molecular assay in cytologically indeterminate thyroid nodules. Endocrine. 2020;68(2):458-65. https://doi.org/10.1007/s12020-020-02271-y 